\title{
Understanding the landscape*
}

\author{
Michael R. Douglas \\ NHETC, Rutgers University \\ Piscataway, NJ 08855-0849, USA \\ and I.H.E.S., Le Bois-Marie, Bures-sur-Yvette, 91440 France \\ mrd@physics.rutgers.edu
}

August 10, 2018

\section{Historical analogies}

At a meeting such as this, one is tempted to compare our present struggles to understand string theory, and to find clearer evidence for or against the claim that it describes our universe, with the deep issues discussed at past Solvay conferences. Now my experience has been that when visiting Belgium, giving in to temptation is generally a good thing to do, so with no further apology let us proceed.

As was beautifully described here by Peter Galison, the 1911 meeting focused on the theory of radiation, and the quantum hypotheses invented to explain black body radiation and the photoelectric effect. These were simple descriptions of simple phenomena, which suggested a new paradigm. This was to accept the basic structure of previous models, but modify the laws of classical mechanics by inventing new, somewhat ad hoc rules governing quantum phenomena. This paradigm soon scored a great success in Bohr's theory of the hydrogen atom. The discovery of the electron and Rutherford's scattering experiments had suggested modeling an atom as analogous to a planetary system. But while planetary configurations are described by continuous parameters, real atoms have a unique ground state, well-defined spectral lines

*Based on comments made at the 23rd Solvay Conference, December 2005, Brussels 
associated with transitions from excited states, etc. From Bohr's postulate that the action of an allowed trajectory was quantized, he was able to deduce all of these features and make precise numerical predictions.

While very successful, it was soon found that this did not work for more complicated atoms like helium. A true quantum mechanics had to be developed. Most of its essential ideas had appeared by the 1927 meeting. Although the intuitions behind the Bohr atom turned out to be correct, making them precise required existing but unfamiliar mathematics, such as the theories of infinite dimensional matrices, and wave equations in configuration space.

Are there fruitful analogies between these long-ago problems and our own? What is the key issue we should discuss in 2005? What are our hydrogen atom(s)?

If we have them, they are clearly the maximally supersymmetric theories, whose basic physics was elucidated in the second superstring revolution of 1994-98. It's too bad we can't use them to describe real world physics. But they have precise and pretty formulations, and can be used to model one system we believe exists in our universe, the near-extremal black hole. We now have microscopic models of black holes, which explain their entropy.

Perhaps we can place our position as analogous to the period between 1913 and $1927 .^{1}$ Starting from our simple and attractive maximally supersymmetric theories, we are now combining their ingredients in a somewhat ad hoc way, to construct $N=1$ and nonsupersymmetric theories, loose analogs of helium, molecules, and more complicated systems. The Standard Model, with its 19 parameters, has a complexity perhaps comparable to a large atom or small molecule. The difficulty of our present struggles to reproduce its observed intricacies and the underlying infrastructure (moduli stabilization, supersymmetry breaking), discussed here by Kallosh, Lüst and others, are probably a sign that we have not yet found the best mathematical framework.

\section{The chemical analogy}

What might this "best mathematical framework" be? And would knowing it help with the central problems preventing us from making definite predictions and testing the theory?

\footnotetext{
${ }^{1}$ A similar analogy was made by David Gross in talks given around 2000. However, to judge from his talk here, he now has serious reservations about it.
} 
In my opinion, the most serious obstacle to testing the theory is the problem of vacuum multiplicity. This has become acute with the recent study of the string/M theory landscape. We have a good reason to think the theory has more than $10^{122}$ vacua, the Weinberg-Banks-Abbott-Brown-TeitelboimBousso-Polchinski et al solution to the cosmological constant problem. Present computations give estimates more like $10^{500}$ vacua. We do not even know the number of candidate vacua is finite. Even granting that it is, the problem of searching through all of them is daunting. Perhaps a priori selection principles or measure factors will help, but there is little agreement on what these might be. We should furthermore admit that the explicit constructions of vacua and other arguments supporting this picture, while improving, are not yet incontrovertible.

We will shortly survey a few mathematical frameworks which may be useful in coming to grips with the landscape, either directly or by analogy. They are generally not familiar to physicists. I think the main reason for this is that analogous problems in the past were attacked in different, nonmathematical ways. Let us expand a bit on this point.

String theory is by no means the first example of an underlying simple and unique framework describing a huge, difficult to comprehend multiplicity of distinct solutions. There is another one, very well known, which we might consider as a source of analogies.

As condensed matter physicists never tire of reminding us, all of the physical properties of matter in the everyday world, and the diversity of chemistry, follow in principle from a well established "theory of everything," the Schrödinger equations governing a collection of electrons and nuclei. Learning even the rough outlines of the classification of its solutions takes years and forms the core of entire academic disciplines: chemistry, material science, and their various interdisciplinary and applied relatives.

Of course, most of this knowledge was first discovered empirically, by finding, creating and analyzing different substances, with the theoretical framework coming much later. But suppose we were given the Schrödinger equation and Coulomb potential without this body of empirical knowledge? Discovering the basics of chemistry would be a formidable project, and there are many more layers of structure to elucidate before one would reach the phenomena usually discussed in condensed matter physics: phase transitions, strong correlations, topological structures and defects, and so on.

As in my talk at String 2003, one can develop this analogy, by imagining beings who are embedded in an effectively infinite crystal, and can only do 
low energy experiments. Say they can observe the low-lying phonon spectrum, measure low frequency conductivity, and so on. Suppose among their experiments they can create electron-hole bound states, and based on phenomenological models of these they hypothesize the Schrödinger equation. They would have some empirical information, but not the ability to manipulate atoms and create new molecules. How long would it take them to come up with the idea of crystal lattices of molecules, and how much longer would it take them to identify the one which matched their data?

Now, consider the impressive body of knowledge string theorists developed in the late 1990's, assembling quasi-realistic compactifications out of local constituents such as branes, singularities, and so on. Individual constituents are simple, their basic properties largely determined by the representation theory of the maximal supersymmetry algebras in various dimensions. The rules for combining pairs of objects, such as intersecting branes or branes wrapping cycles - which combinations preserve supersymmetry, and what light states appear - are not complicated either. What is complicated is the combination of the whole required to duplicate the Standard Model, stabilize moduli, break supersymmetry and the rest. Perhaps all this is more analogous to chemistry than we would like to admit.

Other parallels can be drawn. For example, ${ }^{2}$ according to standard nuclear physics, the lowest energy state of a collection of electrons, protons and neutrons is a collection of ${ }^{62} \mathrm{Ni}$ atoms, and thus almost all molecules in the real world are unstable under nuclear processes. Suppose this were the case for our crystal dwellers as well. After learning about these processes, they might come to a deep paradox: how can atoms other than nickel exist at all? Of course, because of Coulomb barriers, the lifetime of matter is exceedingly long, but still finite, just as is claimed for the metastable de Sitter vacua of KKLT.

Perhaps all this is a nightmare from which we will awake, the history of Kekulé's dream being repeated as farce. If so, all our previous experience as physicists suggests that the key to the problem will be to identify some sort of simplicity which we have not seen in the problem so far. One might look for it in the physics of some dual or emergent formulation. But one might also look for it in mathematics. It is not crazy to suppose that the only consistent vacua are those which respect some principle or have some property which would only be apparent in an exact treatment. But what is

\footnotetext{
${ }^{2}$ As recalled here by Joe Polchinski.
} 
that exact treatment going to look like? The ones we have now cannot be formulated without bringing in mathematics such as the geometry of CalabiYau manifolds, or the category theory underlying topological string theory. If we ever find exact descriptions of $N=1$ or broken supersymmetry vacua, surely this will be by uncovering even more subtle mathematical structures.

But suppose the landscape in its present shape is real, and the key to the problem is to manage and abstract something useful out of its complexity. The tools we will need may not be those we traditionally associated with fundamental physics, but might be inspired by other parts of physics and even other disciplines. But such inspiration can not be too direct; the actual problems are too different. Again, we are probably better off looking to mathematical developments which capture the essence of the ideas and then generalize them, as more likely to be relevant.

On further developing these analogies, one realizes that we do not know even the most basic organizing principles of the stringy landscape. For the landscape of chemistry, these are the existence of atoms, the maximal atomic number, and the facts that each atom (independent of its type) takes up a roughly equal volume in three-dimensional space and that binding interactions are local. These already determine the general features of matter, such as the fact that densities of solids range from $1-20 \mathrm{~g} / \mathrm{cm}^{3}$. Conjectures on the finite number of string vacua, on bounds on the number of massless fields or ranks of gauge groups, and so on, are suggestions for analogous general features of string vacua. But even knowing these, we would want organizing principles. The following brief overviews should be read with this question in mind.

\section{Two-dimensional CFT}

This is not everything, but a large swathe through the landscape. We do not understand it well enough. In particular, the often used concept of "the space of 2d CFT's," of obvious relevance for our questions, has never been given any precise meaning.

A prototype might be found in the mathematical theory of the space of all Riemannian manifolds. This exists and is useful for broad general statements. We recall Cheeger's theorem [5]:

A set of manifolds with metrics $\left\{X_{i}\right\}$, satisfying the following bounds,

1. diameter $\left(X_{i}\right)<d_{\max }$ 
2. Volume $\left(X_{i}\right)>V_{\min }$

3. Curvature $K$ satisfies $\left|K\left(X_{i}\right)\right|<K_{\max }$ at every point,

contains a finite number of distinct homeomorphism types (and diffeomorphism types in $D \neq 4)$.

Since (2) and (3) are conditions for validity of supergravity, while (1) with $d_{\max } \sim 10 \mu \mathrm{m}$ follows from the validity of the gravitational inverse square law down to this distance, this theorem implies that there are finitely many manifolds which can be used for candidate supergravity compactifications [9. 2]

This and similar theorems are based on more general quasi-topological statements such as Cheeger-Gromov precompactness of the space of metrics - i.e., infinite sequences have Cauchy subsequences, and cannot "run off to infinity." This is shown by constructions which break any manifold down into a finite number of coordinate patches, and showing that these patches and their gluing can be described by a finite amount of data.

Could we make any statement like this for the space of CFT's? (a question raised by Kontsevich). The diameter bound becomes a lower bound $\Delta_{\min }$ on the operator dimensions (eigenvalues of $L_{0}+\bar{L}_{0}$ ). We also need to fix $c$. Then, the question seems well posed, but we have no clear approach to it. Copying the approach in terms of coordinate patches does not seem right.

The key point in defining any "space" of anything is to put a topology on the set of objects. Something less abstract from which a topology can be derived is a distance between pairs of objects $d(X, Y)$ which satisfies the axioms of a metric, so that it can be used to define neighborhoods.

The usual operator approach to CFT, with a Hilbert space $\mathcal{H}$, the Virasoro algebras with $H=L_{0}+\bar{L}_{0}$, and the operator product algebra, is very analogous to spectral geometry:

$$
\begin{gathered}
L_{0}+\bar{L}_{0} \text { eigenvalues } \sim \text { spectrum of Laplacian } \Delta \\
\text { o.p.e. algebra } \sim \text { algebra of functions on a manifold }
\end{gathered}
$$

Of course the o.p.e. algebra is not a standard commutative algebra and this is analogy, but a fairly close one.

A definition of a distance between a pair of manifolds with metric, based on spectral geometry, is given in Bérard, Besson, and Gallot [4. The idea is to consider the entire list of eigenfunctions $\psi_{i}(x)$ of the Laplacian,

$$
\Delta \psi_{i}=\lambda_{i} \psi_{i}
$$


as defining an embedding $\Psi$ of the manifold into $\ell_{2}$, the Hilbert space of semi-infinite sequences (indexed by $i$ ):

$$
\Psi: x \rightarrow\left\{e^{-t \lambda_{1}} \psi_{1}(x), e^{-t \lambda_{2}} \psi_{2}(x), \ldots, e^{-t \lambda_{n}} \psi_{n}(x), \ldots\right\}
$$

We weigh by $e^{-t \lambda_{i}}$ for some fixed $t$ to get convergence in $\ell_{2}$.

Then, the distance between two manifolds $M$ and $M^{\prime}$ is the Hausdorff distance $d$ between their embeddings in $\ell_{2}$. Roughly, this is the amount $\Psi(M)$ has to be "fuzzed out" to cover $\Psi\left(M^{\prime}\right)$.

In principle this definition might be directly adapted to CFT, where the $x$ label boundary states $|x\rangle$ (which are the analog of points) and the $\psi_{i}(x)$ are their overlaps with closed string states $\left|\phi_{i}\right\rangle$,

$$
x \rightarrow\left\langle\phi_{i}\left|e^{-t\left(L_{0}+\bar{L}_{0}\right)}\right| x\right\rangle .
$$

Another candidate definition would use the o.p.e. coefficients

$$
\phi_{i} \phi_{j} \rightarrow \sum C_{i j}^{k}\left(z_{i}-z_{j}\right)^{\Delta_{k}-\Delta_{i}-\Delta_{j}} \phi_{k}
$$

for all operators with dimensions between $\Delta_{\min }$ and some $\Delta_{\max }$ (one needs to show that this choice drops out), again weighted by $e^{-t\left(L_{0}+\bar{L}_{0}\right)}$. The distance between a pair of CFT's would then be the $\ell_{2}$ norm of the differences between these sets of numbers.

While abstract, this would make precise the idea of the "space of all $2 \mathrm{D}$ CFT's" and give a foundation for mapping it out.

\section{Topological open strings and derived cate- gories}

This gives an example in which we actually know "the space of all X" in string theory. It is based on the discussion of boundary conditions and operators in CFT, which satisfy an operator product algebra with the usual non-commutativity of open strings. If we modify the theory to obtain a subset of dimension zero operators (by twisting to get a topological open string, taking the Seiberg-Witten limit in a $B$ field, etc.), the o.p.e. becomes a standard associative but non-commutative algebra. This brings us into the realm of noncommutative geometry.

There are many types of noncommutative geometry. For the standard topological string obtained by twisting an $N=2$ theory, the most appropriate 
is based on algebraic geometry. As described at the Van den Bergh 2004 Francqui prize colloquium, this is a highly developed subject, which forms the backdrop to quiver gauge theories, D-branes on Calabi-Yau manifolds, and so on.

One can summarize the theory of D-branes on a Calabi-Yau $X$ in these terms as the "Pi-stable objects in the derived category $D(\operatorname{Coh} X)$," as reviewed in [3]. Although abstract, the underlying idea is simple and physical. It is that all branes can be understood as bound states of a finite list of "generating branes," one for each generator of $\mathrm{K}$ theory, and their antibranes. The bound states are produced by tachyon condensation. Varying the CalabiYau moduli can vary masses of these condensing fields, and if one goes from tachyonic to massive, a bound state becomes unstable.

This leads to a description of all D-branes, and "geometric" pictures for all the processes of topology change which were considered "non-geometric" from the purely closed string point of view. For example, in a flop transition, an $S^{2} \Sigma$ is cut out and replaced with another $S^{2} \Sigma^{\prime}$ in a topologically different embedding. In the derived category picture, what happens is that the brane wrapped on $\Sigma$, and all D0's (points) on $\Sigma$, go unstable at the flop transition, to be replaced by new branes on $\Sigma^{\prime}$.

The general idea of combining classical geometric objects, using stringy rules of combination, and then extrapolating to get a more general type of geometry, should be widely useful.

\section{Computational complexity theory}

How hard is the problem of finding quasi-realistic string vacua? Computer scientists classify problems of varying degrees of difficulty:

- P can be solved in time polynomial in the size of the input.

- An NP problem has a solution which can be checked in polynomial time, but is far harder to find, typically requiring a search through all candidate solutions.

- An NP-complete problem is as hard as any NP problem - if any of these can be solved quickly, they all can.

It turns out that many of the problems arising in the search for string vacua are in NP or even NP-complete. 6] For example, to find the vacua in 
the Bousso-Polchinski model with cosmological constant $10^{-122} M_{\text {Planck }}^{4}$, one may need to search through $10^{122}$ candidates.

How did the universe do this? We usually say that the "multiverse" did it - many were tried, and we live in one that succeeded. But some problems are too difficult for the multiverse to solve in polynomial time. This is made precise by Aaronson's definition of an "anthropic computer." [1]

Using these ideas, Denef and I [7] have argued that the vacuum selected by the measure factor $\exp 1 / \Lambda$ cannot be found by a quantum computer, working in polynomial time, even with anthropic postselection. Thus, if a cosmological model realizes this measure factor (and many other preselection principles which can be expressed as optimizing a function), it is doing something more powerful than such a computer.

Some cosmological models (e.g. eternal inflation) explicitly postulate exponentially long times, or other violations of our hypotheses. But for other possible theories, for example a field theory dual to eternal inflation, this might lead to a paradox.

\section{Conclusions}

We believe string theory has a set of solutions, some of which might describe our world. Even leaving aside the question of few vacua or many, and organizing principles, perhaps the most basic question about the landscape is whether it will turn out to be more like mathematics, or more like chemistry.

Mathematical analogy: like classification of Lie groups, finite simple groups, Calabi-Yau manifolds, etc. Characterized by simple axioms and huge symmetry groups. In this vision, the overall structure is simple, while the intricacies of our particular vacuum originate in symmetry breaking analogous to that of more familiar physical systems.

Chemical analogy: simple building blocks (atoms; here branes and extended susy gauge theory sectors) largely determined by symmetry. However, these are combined in intricate ways which defy simple characterization and require much study to master.

The current picture, as described here by Kallosh and Lüst, seems more like chemistry. Chemistry is a great science, after all the industrial chemistry of soda is what made these wonderful conferences possible. But it will surely be a long time (if ever) before we can manipulate the underlying constituents of our vacuum and produce new solutions, so this outcome would be less 
satisfying.

Still, our role as physicists is not to hope that one or the other picture turns out to be more correct, but to find the evidence from experiment and theory which will show us which if any of our present ideas are correct.

Acknowledgments I would like to thank the organizers and the Solvay Institute for a memorable meeting, and B. Acharya, F. Denef, M. Kontsevich, S. Zelditch and many others for collaboration and discussion of these ideas. This research was supported in part by DOE grant DE-FG02-96ER40959.

\section{References}

[1] S. Aaronson, "Quantum Computing, Postselection, and Probabilistic Polynomial-Time," arXiv:quant-ph/0412187.

[2] B. Acharya and M. R. Douglas, to appear.

[3] P. S. Aspinwall, "D-branes on Calabi-Yau manifolds," arXiv:hep-th/0403166.

[4] P. Bérard, G. Besson, and S. Gallot, "Embedding Riemannian manifolds by their heat kernel," Geom. Funct. Anal. 4 (1994), 373-398.

[5] J. Cheeger, "Finiteness theorems for Riemannian manifolds," Am. J. Math 92 (1970), 61-74.

[6] F. Denef and M. R. Douglas, "Computational Complexity of the Landscape I," hep-th/0602072.

[7] F. Denef and M. R. Douglas, "Computational Complexity of the Landscape II," to appear.

[8] M. R. Douglas, talk at Strings 2003.

[9] M. R. Douglas, talk at Strings 2005. 